A RTIGOS TrRADUZIDOS/
TransLATED A RTICLES 


\title{
A TRADUÇÃO DO TEATRO ENQUANTO COLABORAÇÃO: UM CASO EM QUESTÃO NO DRAMA BRITÂNICO CONTEMPORÂNEO ${ }^{1}$
}

\author{
Andrea Peguinelli* \\ Università di Roma La Sapienza \\ Tradução de: Andreza Sara Caetano de Avelar Moreira** \\ Universidade Federal de Minas Gerais
}

\begin{abstract}
Resumo: A tradução de teatro é geralmente vista como uma dimensão mais elaborada da tradução literária, porque o texto a ser traduzido é considerado apenas um dos elementos do discurso de teatro. Ao traduzir uma peça, o tradutor deve sempre adaptá-lo ao desempenho do texto que está recriando e estar ciente de que um ator vai proferir as linhas. O tradutor, então, deve levar em conta tanto a pragmática quanto a expressividade semântica da palavra e lembrar-se que ambas funcionam sempre simultaneamente. Tomarei exemplos tanto da minha experiência pessoal quanto de casos notáveis, analisarei como uma boa tradução pode afetar a recepção do público de uma peça que lhe é estranha, e mostrarei que é
\end{abstract}

\footnotetext{
* Andrea Peguinelli possui diploma em Tradução Literária e em línguas e literaturas modernas e contemporâneas e doutorado em Literatura de Língua inglesa pela Università di Roma La Sapienza. Atualmente é professora da Università di Roma La Sapienza.. Roma, Itália. E-mail: andrea.peghinelli@uniroma1.it

** Andreza Caetano, tradutora deste artigo, possui bacharelado em Grego Clássico pela Universidade Federal de Minas Gerais. É também licenciada pelo Centro Universitário Claretiano em Português/Inglês. Possui mestrado em Estudos Literários pela Universidade Federal de Minas Gerais (Estudos Clássicos, Poéticas da Tradução) sob orientação de Tereza Virginia Ribeiro Barbosa. Belo Horizonte, Minas gerais, Brasil. E-mail: andrezacaetano@yahoo.com.br
} 
primeiramente através de uma abordagem pragmática que se torna possível obter a consciência de qual é a forma mais adequada de se interpretar o texto original.

Palavras-chave: Tradução de teatro. Drama Inglês contemporâneo. Abordagem pragmática. Contexto holístico.

\title{
THEATRE TRANSLATION AS COLLABORATION: A CASE IN POINT IN BRITISH CONTEMPORARY DRAMA
}

\begin{abstract}
Theatre translation is usually seen as a more elaborate dimension of literary translation because the text being translated is considered to be just one of the elements of theatre discourse. When translating a play, the translator should always adapt for performance the text he or she is recreating and be aware that a performer will deliver the lines. The translator, then, must take into account both the pragmatic and the semantic expressiveness of the word and remember that they are always at work simultaneously. I will take examples both from my personal experience and from remarkable cases in point of how a good translation may affect an audience reception of a foreign play and I will show that it is primarily through a pragmatic approach that it is possible to obtain an awareness of what is the most appropriate way of rendering the original text.
\end{abstract}

Keywords: Theatre translation. Contemporary English drama. Pragmatic approach. Holistic context.

Uma possível maneira de olhar para a atividade de tradução pode ser a considerando-a um espelho que é erguido até representar fielmente um texto de origem. A imagem refletida nunca será exatamente o mesmo que o original; no entanto, é para ser confiável como uma verdadeira cópia dele. A melhor realização, então, que podemos visar ao traduzir é "dizer quase a mesma coisa", como Umberto Eco afirmou no título de seu extenso estudo sobre tradução ${ }^{2}$. Embora este processo de representação textual de uma língua em outra possa levar à conclusão de que é uma tarefa que não poderia ser totalmente cumprida, em um paradoxo, eu gostaria de afirmar que, por outro lado, está longe de ser um fracasso absoluto. Devemos ler a tradução à luz da estética do fracasso que Samuel 
Beckett aderiu em Worstward Ho: se a linguagem, por definição, falha em traduzir o pensamento, ela ainda é cúmplice em se tratando de representá-1o3; portanto, se estamos conscientes de que não é possível traduzir "fielmente" um texto original em outra língua, ainda assim a tradução materializa essa impossibilidade. Apesar de sabermos que estamos condenados ao fracasso, essa consciência deve gerar uma expansão na atividade criativa. Afinal, tal como escreveu Beckett, o que todo artista pode fazer é reiterar suas tentativas: "Já tentou. Nunca falhou. Não importa. Tente novamente. Falhe novamente. Fracasse melhor" ${ }^{4}$. Desde que li isso, tem se tornado meu lema como um tradutor.

A tradução do teatro é geralmente vista como uma dimensão mais complexa da tradução literária, já que o texto a ser traduzido é pensado para ser apenas um dos elementos do discurso de teatro que se deve processar em uma diferente língua. Além disso, o teatro é um espelho do mundo, um espelho que não só reflete as expressões verbais, mas também as ações, os gestos, os silêncios, todo o aparato que acompanha. É por isso que na tradução para o teatro a impossibilidade intrínseca da tradução torna-se um processo ainda mais complicado. No entanto, como Anne Ubersfeld escreveu em Reading Theatre: "No teatro o impossível reina, o teatro trabalha com o impossível, como uma forma de expressar o impossível." 5 Como questão de fato, a figura retórica prevalecente no teatro é o paradoxo que, por exemplo, mostra as contradições enraizadas nos personagens - e na situação na qual eles têm que lidar - que fazem as ações teatrais se moverem no palco. ${ }^{6}$

"O drama, como uma forma de arte, é um constante processo de tradução", como Reba Gonstand observou, e "desde o conceito original de roteiro (quando há um), a interpretação do diretor ou do produtor, a contribuição do designer e do ator, do visual e/ou das imagens aurais, da resposta do público [ ... ] pode haver uma série de processos auxiliares de tradução no trabalho." 7 Na recriação dinâmica de um texto de origem e, em seguida, com a consciência da peculiaridade de uma linguagem estilística, das diferenças culturais e diferentes convenções teatrais, devemos também ter em mente 
que, no ato conseqüente de encenar uma peça sempre há diferentes tipos de traduções envolvidos. Podemos, então, afirmar que o texto escrito é incompleto, uma vez que está indissoluvelmente ligado à performance - como Ubersfeld afirmou que é um " troué (buraco)", tem lacunas ${ }^{8}$ - por isso temos que preencher os espaços em branco, encontrando respostas no próprio desempenho, e só esta combinação nos dá um texto teatral completo, um texto mediado pelos dois elementos. Ela argumenta que a distinção entre texto escrito e performance é artificial, uma vez que os dois não podem ser separados. Seu relacionamento não é apenas o de uma equivalência semântica porque o desempenho não pode ser entendido como uma mera "tradução" do texto. Seria perigoso supor que a expressão de um contexto resulta de um sistema de conversão do signo linguístico para um sistema de signos performáticos ou de palco; de fato, esta posição poderia nos levar à conclusão de que existe apenas uma única e correta maneira de interpretá-la.

Sabemos que diferentes performances nos dão diferentes leituras de um texto, e não é só uma questão de escolha de um diretor, porque é mesmo impossível reproduzir performaticamente o mesmo texto noite após noite: notaremos diferentes gestos, pequenas mudanças na entonação, diferentes respostas de e para um público diferente presente no auditório. Como consequência, pode-se inferir que não há um texto definitivo. No entanto, embora pareça incompleto, é apenas através da sua estrutura que o aspecto performativo pode se expressar, e é a matéria-prima sobre o qual o tradutor deve trabalhar. Como Susan Bassnet observou, "é com o texto escrito, e não com um desempenho hipotético, que o tradutor deve começar." No entanto, o tradutor também sabe que a tradução não pode manter o texto em seu estado original, pois se destina a uma situação futura de enunciação encenada para um público-alvo real. Portanto, como Patrice Pavis comentou, a tradução ocorre no cruzamento das situações de enunciação em uma determinada língua da cultura alvo; e mesmo que tenhamos apenas uma vaga noção de uma determinada cultura, inevitavelmente, é dentro dos aspectos que determinam a sua identidade que a tradução ocorre: 
A tradução é este texto mítico não detectável que tenta levar em conta o texto de origem - o tempo todo com a consciência de que tal texto só existe como referência a uma fonte texto-a-ser-traduzido. Somado a essa circularidade preocupante está o fato de que a tradução de teatro nunca está onde se espera que esteja: não em palavras, mas em gestos; não na letra, mas no espírito de uma cultura, inefável, mas onipresente. ${ }^{10}$

Ao traduzir uma peça, o tradutor deve sempre adaptar o texto que ele ou ela está recriando para a performance e estar ciente de que um ator vai proferir as linhas. Claro o suficiente, então, sua tarefa não é simplesmente decodificar um texto em um idioma diferente criando uma equivalência linguística. Na verdade, acredito que não existe algo como uma tradução literal, porque ela vai sempre ser o produto de alguém que recria um texto para a performance, a fim de conceder acesso público a um texto específico. Afinal, uma tradução consiste em mudar um texto para atender às diferentes necessidades. O ofício do tradutor só vai produzir um texto transitório, enquanto a obra de arte é atemporal. O contexto sócio-cultural, tão incondicionalmente claro para o público contemporâneo e para os concidadãos, ainda é vazio ou simplesmente ornamental para os retardatários históricos ou os que estão fora dele: o sentido perdido, precisamos recuperar pela substituição de detalhes do texto-fonte por elementos que são comunicativos para nós; da mesma forma como os da peça original eram para o público fonte. ${ }^{11}$ O tradutor, então, deve sentir tanto a pragmática quanto a expressividade semântica da palavra, observando as tradições dramáticas distintas dos idiomas de e para o qual está traduzindo. A tradução do teatro acima de tudo tem que funcionar dentro do contexto imediato da performance - sem anotações ou comentário editorial.

Muitas vezes, o tradutor também é solicitado para atender às expectativas de um público específico e fornecer detalhes familiares para ele na nova versão. Esta estratégia pode levar a adaptações que têm uma grande distância do original e, eventualmente, pode 
sofrer a influência pesada do diretor ou do produtor, que impõe suas próprias escolhas.

Então, quais são as estratégias que devem ser desenvolvidas para lidar com o que parece ser intraduzível ou indizível? O que precisamos é, provavelmente, de uma combinação de processos de adaptação, interpretação, paráfrases, contemporização, e o mais importante, compreensão e colaboração. É este contexto holístico, como sugere Andras Nagy, mais que um texto distinto, o que representa o verdadeiro desafio para o tradutor de teatro. ${ }^{12} \mathrm{E}$ sua observação provavelmente concede um "verdadeiro respeito" para o texto a ser traduzido. ${ }^{13}$

Se olharmos novamente para o símile do espelho, a tradução peculiar adaptada para funcionar em um país pode ser apenas um fragmento do espelho quebrado em que a imagem original foi refletida. Daí resulta que um texto teatral traduzido surge como uma variedade de reflexões e imagens geradas pelo contexto histórico e pelos ambientes culturais, como se estivéssemos olhando para o original através de um caleidoscópio.

A recriação ideal da peça original não deve apenas transmitir suas características linguísticas e suas referências culturais, mas também deve procurar reproduzir ou "sabiamente" interpretar os aspectos decorrentes do processo colaborativo que o produziu. Isto é particularmente verdadeiro para aquelas peças nas quais dramaturgos têm feito oficinas na estreita em colaboração com os diretores e atores. Na tradução, parece haver uma relação de assimetria com o texto original; pois este não é distorcido, mas deliberadamente re-trabalhado para enfrentar o efêmero momento em que, finalmente, será performatizado ${ }^{14}$. $\mathrm{O}$ tradutor, como re-criador do texto, deve ser reconhecido como uma figura-chave no processo colaborativo da produção, comparável ao papel do dramaturgo do dramaturgo e do diretor. Cada nova tradução, como cada nova produção, envolve um conjunto distinto de escolhas artísticas e pragmáticas, o resultado não pode pretender ser definitivo, ele só funciona até que a voz através do qual se fala tem ressonância para o seu público-alvo. 
O tradutor raramente tem sido reconhecido como uma figura criativa e parte integrante do processo de produção, uma figura criativa na elaboração do texto performático através de ensaios e reuniões de produção; como, aliás, a prática corrente na Itália, por exemplo, onde traduzir textos contemporâneos cabe a uma comissão de tradução composta por acadêmicos ou especialistas em uma determinada língua estrangeira para se obter um texto bruto que pode ser reformulado em uma nova versão de um conhecido dramaturgo - porém, mais frequentemente, pelo próprio diretor, mesmo que ele nem sempre tenha a necessária competência na língua de origem (esta prática surge muitas vezes mais do que uma suspeita sobre o hábito desprezível de plagiar traduções anteriores do texto em questão). Com clássicos, uma tradução publicada anteriormente geralmente é readaptada pelo próprio diretor. Isso só acontece quando o tradutor não é uma figura de destaque na cena cultural ou quando não é o tradutor oficial de um dramaturgo particular; e, provavelmente, porque as novas versões ou adaptações parecem ter um status mais elevado do que as traduções ${ }^{15}$. Talvez a implicação seja de que uma adaptação requer um nível de habilidade dramatúrgica e visão criativa, que, por uma mera tradução, é exclusivamente atribuível ao dramaturgo original? Esta sugestão provocante transmite alguma verdade: muitas vezes os tradutores não são treinados para traduzir para o teatro. Na maioria dos casos, eles só têm uma formação acadêmica literária.

\section{Um caso em questão}

Minha primeira experiência pessoal de colaboração no processo criativo de tradução é um bom exemplo para ir mais longe nesta exploração. O texto que vou levar em consideração é de Philip Ridley The Pitchfork Disney. Eu me deparei com ele ao traduzir In-Yer-Face Theatre de Aleks Sierz em italiano. Foi uma experiência muito proveitosa, pois me permitiu mergulhar, literalmente, em um drama britânico contemporâneo e adquirir um conhecimento 
profundo da inovação teatral que teve lugar no final do século 20 . Além disso, as respostas rápidas de Aleks às minhas perguntas foram cruciais para me dar confiança para completar a tradução com o conhecimento que eu estava servindo o propósito dele. Dicas de um autor são sempre úteis para um tradutor - não importa qual o gênero do texto no qual você está trabalhando.

Fiquei fascinado com a peça de Ridley, um dos primeiros a introduzir uma nova sensibilidade na cena do teatro de Londres ${ }^{16}$. Quando, em 2005, juntamente com o grupo que formou com outros ex-alunos da escola de teatro que participei, fomos convidados a encenar uma peça de teatro em um festival de teatro em Spoleto I, não hesitou em propor a Pitchfork Disney. Poderíamos ter trabalhado com uma tradução existente da peça, publicada como $O$ assassino da Disney, mas eu não gostava dela por causa de sua literalidade, a sua falta de ritmo teatral, sintaxe elaborada e seu servilismo; por isso, resolvi traduzi-la novamente. Minha educação profissional como tradutor é principalmente acadêmica, mas eu fui ensinado por professores que muitas vezes trabalharam no teatro como tradutores ou diretores. Além disso, sendo eu mesmo também um ator, eu poderia me aproximar do texto escrito com ouvido para a performance.

Quando um primeiro esboço da tradução estava pronto, perguntei a uma dramaturga (que estudou atuação comigo) para lê-lo e fazer os ajustes que acreditava necessários. Ela provavelmente foi muito criativa. Como resultado, re-imaginado o texto através dos olhos da protagonista feminina, mudou muita coisa: Tive que lutar para restaurar o que eu achava que era necessário. Não obstante, ela também introduziu algumas soluções muito interessantes do ponto de vista semântico, e o texto adquiriu uma dimensão coloquial que melhorou a franqueza da narrativa da história. No final, ela interpretou a protagonista feminina tornando-se perfeitamente sintonizada com esse papel. Eu interpretei o papel principal masculino. E trabalhando juntos, nos ajudamos muito determinando o tom da tradução com o registro "correto", apesar de a peça ter sido montada no East End de Londres, que não tinha qualquer preocu- 
pação verdadeira com dialeto e classe de língua flexionada. Nós apenas diferenciamos da linguagem infantil de Haley e Presley, os dois irmãos que estávamos encenando, a partir de uma espécie de jargão utilizado pelo Cosmo: o artista, um personagem que é o seu oposto. O processo de adaptação foi revelador de muitos aspectos da interpretação teatral, pelo que a ação tornou-se mais falante do que o originalmente planejado. Além disso, ao invés de um ambiente naturalista, o diretor escolheu encenar com apenas alguns elementos do mobiliário: o esqueleto de uma porta e de uma janela, uma geladeira velha e um par de cadeiras, que eram constantemente movidos em cena para enfatizar alterações nos desenvolvimentos da perspectiva dos personagens.

$\mathrm{Eu}$ estava ciente do público para a qual deveríamos encenar. Geralmente em festivais você tem um público esperto em teatro, que tem amplo conhecimento da cena teatral. Então, decidi não traduzir alguns elementos (ou seja, o título e os nomes dos protagonistas) e as referências ao anglicismo do jogo britânico (por exemplo, xícaras de chá). Além disso, após a recente onda de nova redação proveniente do Reino Unido, uma tendência que nós esperávamos explorar comercialmente - tinha sido criada para peças britânicas. No entanto, não acredito que mudar totalmente o material de origem estrangeira dentro da experiência cultural do público-alvo teria ajudado na compreensão da peça. Se um processo de domesticação representa uma traição indevida da fonte, ou o devido reconhecimento do alvo, ainda é uma questão de opinião. A decisão de mudar uma peça é, sem dúvida, mais consequente, visando um texto para o desempenho; mas isso ainda é uma decisão a ser tomada com escrupulosa atenção. E o tradutor deve estar bem consciente do constante processo de redefinição da cultura alvo contemporânea.

Depois de encenar ao ar livre, em Spoleto, em um claustro encantador do século XIV e na frente de uma platéia de cerca de trezentas pessoas, apresentamos $O$ Pitchfork Disney em Roma, em um pequeno teatro de margem, a clássica caixa-preta, por um período de três semanas corridas para uma audiência média de cerca de 
trinta pessoas. Como consequência, tivemos que voltar a trabalhar na tradução. É muito diferente quando você pronuncia uma linha e tem que caminhar por sete ou oito metros, ou se você apenas tem que dar dois ou três passos. O texto muda, o espaço muda, a atuação muda. Tentei modular as palavras em um tom mais adequado para o espaço; condensar as linhas, a fim de obter uma linguagem mais básica. Além disso, juntamente com o diretor, cortamos o texto. Pensamos que alguns dos monólogos extensos não teriam sido "tolerados" pelo público diferente naquele auditório claustrofóbico. Em seguida, mudamos para um teatro maior em Roma e também fizemos uma pequena turnê, mas não alteramos significativamente o texto depois.

A experiência acima indicada reforçou em mim a crença de que um tradutor ideal para o teatro, não deve ser treinado somente na universidade para ganhar competência linguística e cultural, mas também em teatros e academias de artes dramáticas. Afinal, um tradutor deve escrever para atores como os dramaturgos fazem. De acordo com este ponto de vista - o que de alguma forma é semelhante ao que Robert Corringan afirmou nos anos sessenta sobre tradutores que estão sendo treinados na prática de teatro: "Sem essa formação, a tendência será a de traduzir palavras e seus significados. Esta prática nunca vai produzir traduções encenáveis" 17 -, recentemente promovi um acordo entre a Academia Nacional de Arte Dramática da Itália "Silvio d' Amico" e a "Sapienza” Universidade de Roma, onde leciono cursos de Tradução do Inglês para o Italiano e Drama Inglês (o acordo foi elaborado e assinado durante o ano de 2009/2010 pelo professor acadêmico Isabella Imperiali, em nome da "Sapienza" Universidade de Roma, e pelo mestre Lorenzo Salveti, diretor da Accademia d'Arte Drammatica "Silvio d' Amico"). Os alunos que seguem meus cursos de Tradução para Teatro e do Professor Imperiali serão autorizados a colocar em prática o que eles estudam, e moldar as suas traduções através de uma colaboração com os alunos dos cursos de atuação e direção da Academia. Estudantes da Universidade devem entrar na sala de ensaio do estúdio de teatro da Academia com um primeiro esboço 
de sua tradução (feito sob a minha supervisão) e depois de algumas semanas de ensaios com atores, diretores e professores, eles devem ser capazes de sair com uma tradução que iria responder às exigências do contexto holístico acima referido. Trabalhar em oficinas e interagir cara a cara capacitará os alunos a adquirir a consciência de um método abrangente que ilusoriamente mais tarde será aplicado em suas profissões.

No início de novembro de 2010, tive a oportunidade de colocar em prática o que tinha previsto com o acordo, quando os conselhos da academia e da universidade concordaram em financiar uma oficina sobre as peças de Martin Crimp. Escolhi trabalhar sobre The Treatment, um texto desafiador que Crimp escreveu para o Royal Court Theatre em 1993, cuja tradução ainda não havia sido publicada na Itália. The Treament é uma peça ambientada em Nova York e usa a metrópole como uma convenção, da mesma maneira que alguns dramaturgos jacobinos usaram Veneza para distanciar sua própria sociedade de sua decadência; este drama urbano, como Sierz comentou, é um "grito de consternação no qual a forma é julgada como arte pelo comercial, em vez de pelos critérios estéticos." 18 Selecionei cinco alunos do meu curso de mestrado em tradução e pedi-lhes para traduzir. Atribuí algumas cenas a cada um deles, e quando eles tiveram um primeiro esboço de sua tradução nos encontramos e lemos em voz alta: desta forma, eles foram capazes de confrontar suas soluções e conformar termos recorrentes, nomes, e igualmente transferir metáforas e imagens. A necessidade de compatibilizar os cinco fragmentos diferentes do texto traduzido (um para cada tradutor) era na verdade uma boa maneira de refletir sobre o estilo do dramaturgo. Eu, obviamente, corrigi erros e sugestões propostas para as passagens difíceis, de modo a encontrar, em conjunto, uma solução válida. O mais difícil foi torná-los conscientes da necessidade de encontrar uma forma definitiva para caracterizar o idioleto dos personagens e mantê-lo por toda parte. Conseguimos superar esta dificuldade através de uma análise profunda do texto e um estudo detalhado dos personagens. 
Um ponto importante do projeto foi o encontro que organizamos para os participantes da oficina com Martin Crimp e o crítico de teatro Aleks Sierz. Como, aliás, eu acredito, é extremamente útil ao traduzir um texto de um autor de estilo de vida contemporâneo, ter a possibilidade de fazer perguntas a ele. E ambos, Crimp e Sierz, foram muito generosos com os alunos. Além disso, Crimp traduziu várias peças em Inglês; então, suas sugestões foram realmente úteis e específicas. Ele acha que existem diferentes maneiras de abordar uma tradução para o teatro. É possível ter uma tradução em linha reta a partir de uma linguagem que o tradutor entende, cujo objetivo é fornecer uma janela para o texto original. Neste caso, a única preocupação para o tradutor é que a tradução seja atuável, ele não quer aparecer, mas apenas quer servir ao escritor, à peça e também à produção. A partir de duas traduções, as quais Crimp foi contratado para fazer, podemos inferir duas possibilidades diferentes de proceder neste caso. Quando, em 1997, ele traduziu, de Eugène Ionesco, Les chaises, para o Royal Court Theatre; o diretor Simon McBurney não queria que a peça parecesse um artefato francês, então Crimp suprimiu todas as referências a esse ambiente cultural; mas os manteve ao traduzir do mesmo Ionesco, o Rhinocéros (Royal Court Theatre, 2007), pois o diretor Dominic Cook queria recriar o mundo da França nos anos cinquenta e queria aquele artefato.

Também é possível criar uma tradução direta e transparente com base em um texto intermediário de uma língua que o tradutor não entende. Crimp não gosta desta opção, pois acredita que não nos permite atingir a emoção da língua original. Ele compara a barbear-se diante de um espelho no vapor: você corre o risco de raspar-se muito mal.

Finalmente, um tradutor pode se adaptar, reescrever, renovar e remodelar um texto, de modo a adequá-lo para um público que fala uma língua diferente, vive em um país diferente e em um tempo diferente. Crimp seguiu esse curso de ação quando traduziu Le Misanthrope de Molière (Young Vic, 1996; Comedy Theatre, 2009), porque acredita que, por exemplo, a sátira contingente de 
O Misantropo, se perderia de outra forma. Ele também produziu uma versão mais simples de The Seagull, de Anton Checov (Teatro Nacional, 2006). No texto final, as referências do século XIX foram substituídas por equivalentes, ou completamente removidas, e também se despojou das convenções teatrais do século XIX como monólogos e apartes. A versão de Crimp era obviamente menor, mas também mais vibrante, mais fresca do que o original, renovada, como uma pintura antiga pode ser após um processo de restauração.

Traduzir para a performance dentro de um determinado contexto requer sensibilidade para os vários registros em operação, tanto na cultura-fonte quanto na cultura-alvo - seja em termos de censura do Estado, ou, em um nível mais pragmático, das políticas de produção institucionais. Como defensor cultural, o tradutor pode se recusar a mudar o texto de origem por medo de neutralizar a sua identidade cultural. Por exemplo, quando em 2009 a empresa bielorrussa Belarus Teatro Livre veio encenar na Itália (pelo Vie Scena Contemporanea festival de teatro em Modena), fui convidado a rever e adaptar as traduções de algumas de suas peças, porque eu tinha ido a Minsk para trabalhar com eles, conhecia suas peças e, particularmente, sua condição peculiar de dissidentes do regime totalitário de Alexander Lukashenko. Nesse caso, não houve necessidade de domesticar as peças: o que devia brotar era uma consciência do que é a vida na Bielorrússia sob os abusos de uma ditadura, a qual deveria ser transmitida com consistência absoluta.

\section{Conclusão}

A comunicação intelectual depende sempre de variados e complexos processos que influenciam não só a produção de uma tradução de teatro, mas também a sua distribuição e a recepção de um público-alvo diversificado. É principalmente com prática e com uma abordagem pragmática do que é possível, que se obtém consciência do que é a solução mais adequada de acordo com o lugar e as pes- 
soas envolvidas. No entanto, interessante e estimulante são as duas construções teóricas polarizadas de performabilidade e legibilidade que animaram o debate teórico sobre a tradução para o teatro. Quando aplicadas a traduções reais e performances teatrais elas parecem compartilhar uma fraqueza em suas abordagens prescritivas.

Em particular, acredito que é bastante singular falar sobre a leitura de um texto concebido e escrito para ser falado e realizado. Normalmente, no processo de criação, as palavras que constituem a linguagem da peça são ouvidas primeiro pelo dramaturgo no momento em que seus personagens começam a ganhar vida, ele ou ela, em seguida, os escreve para serem falado por atores e/ou atrizes que por sua vez os entregam para um determinado público que os ouve. Este é mais ou menos o processo do que chamamos de teatro, que é feito, principalmente, por uma combinação de enunciados e ficheiros de som, mesmo que faça uso de palavras escritas. Para ser definida como digna do palco uma tradução deve fluir como os fluxos do texto original. E é isso que uma tradução de teatro deve procurar fazer e o que, em minha opinião, define o bom senso e o julgamento que um tradutor sábio deve ter.

Não tendo nenhum desejo, então, de impor qualquer regra particular ou método em estudos de tradução, só queria que os tradutores falhassem da melhor maneira possível.

\section{Notas}

1. $\mathrm{O}$ artigo, intitulado originalmente Theatre Translation as collaboration: A case in point in British contemporary drama, foi publicado por Journal for Communication and Culture 2, no. 1 (spring 2012). 
2. Cf. Umberto Eco, Dire quasi la stessa cosa, esperienze di traduzione (Milano: Bompiani, 2003).

3. C. J. Ackerley, S. E. Gontarski, eds., The Faber Companion to Samuel Beckett (London: Faber \& Faber, 2006), 652-53.

4. Samuel Beckett, Worstward Ho (London: John Calder, 1983), 7.

5. Anne Ubersfeld, Reading theatre (Toronto: University of Toronto Press, 1999), 190.

6. Cf. Ubersfeld, Reading theatre, $190 \mathrm{ff}$

7. Reba Gonstand, "Verbal and non verbal communication: drama as translation," in O. Zuber ed., The Langage of Theatre. Problems in Translation and Transposition of Drama. (Oxford and New York: Pergamon Press, 1980), 1.

8. Cf. Ubersfeld, Reading theatre, xvi; 10.

9. Susan Bassnet, "Ways through the Labyrinth: Strategies and Methods for Translating Theatre Texts," in Theo Hermans, ed., The Manipulation of Literature (London: Croom Helm; New York: St Martin's, 1985), 102.

10. Patrice Pavis, "Problems of Translation for the Stage: Intercultural and PostModern Theatre," in Peter Holland, Hanna Scolnicov, eds., The Play Out of Context: Transferring Plays from Culture to Culture (Cambridge: Cambridge University Press, 1989), 42.

11. Cf. Andras Nagy, "A samovar is a samovar: Hopes and failures of the author as the object and subject of translation," in Moving Target, Theatre translation and Cultural Relocation, ed. by Carole-Anne Upton (Manchester: St. Jerome Publishing, 2000).

12. Andras Nagy, "A samovar is a samovar," 152.

13. A importância de uma abordagem "holística" foi defendida por Pavis, pelo menos desde 1989, quando, por exemplo, ele escreveu: "O tradutor é um drama- 
turgo, que deve buscar, antes de tudo um efeito de tradução macrotextual, isto é, uma análise dramatúrgica da ficção transmitida pelo texto. Ele deve reconstituir a trama de acordo com a lógica que parece se adequar à ação, e assim reconstituir a totalidade artística ", em Patrice Pavis, "Problems of Translation for the Stage: Intercultural and Post-Modern Theatre," 27.

14. Cf. Hale and Upton, "Introduction," in Moving Target (Manchester, UK \& Northampton MA: St. Jerome Publishing, 2000).

15. Veja, por exemplo, o que diz Maria Delgado states in Phyllis Zatlin, Theatrical translation and film adaptation: a practitioner's view (Clevendon: Multilingual Matters, 2005), 26.

16. Aleks Sierz, In-Yer-Face Theatre: British Drama Today. (London: Faber \& Faber, 2001), 40-47.

17. Robert W. Corrigan, "Translating for Actors," in W. Arrowsmith and R. Shattuck eds., The Craft \& Context of Translation. (Austin: University of Texas Press, 1961), 100.

18. Aleks Sierz, The Theatre of Martin Crimp. (London: Methuen Drama, 2006), 40.

\section{Referências}

Aaltonen, Sirkku. Time Sharing on Stage: Drama Translation in Theatre and Society. Clevendon: Multilingual Matters, 2000.

Corrigan, Robert W. "Translating for Actors," in W. Arrowsmith and R. Shattuck, ed., The Craft \& Context of Translation. Austin: University of Texas Press, 1961. 
Elam, Keir. The Semiotics of Theatre and Drama. London: Routledge, 1980.

Johnston, David. Stages of Translation. London: Absolute Classics, 1996.

Patrice, Pavis. Dictionaire du Théâtre. Paris: Armand Colin, 2002 [1980].

Scolnicov H. and Holland P. The Play out of Context: Transferring Plays from Culture to Culture. Cambridge: Cambridge University Press, 1989.

Venuti, Lawrence. The Translator's Invisibility. London: Routledge, 1995.

Wechsler, Robert. Performing Without a Stage: the Art of Literary Translation. North Haven: Catbird Press, 1998.

Zuber, Ortrun. The Languages of Theatre: Problems in the Translation and Transposition of Drama. Oxford and New York: Pergamon Press, 1980.

Recebido em: 22/09/2014

Aceito em: 03/12/2014 
ARTIGO PUBLICADO ORIGINALMENTE EM:

Journal for Communication and Culture 2, no. 1 (spring 2012): 20-30

${ }^{\odot}$ Institute for Communication and Culture E-ISSN \& ISSN-L: 2247-4404 www.jcc.icc.org.ro•contact@icc.org.ro

\title{
THEATRE TRANSLATION AS COLLABORATION: A CASE IN POINT IN BRITISH CONTEMPORARY DRAMA
}

Andrea Peghinelli

Sapienza University, Department of English Literature

\begin{abstract}
Theatre translation is usually seen as a more elaborate dimension of literary translation because the text being translated is considered to be just one of the elements of theatre discourse. When translating a play, the translator should always adapt for performance the text he or she is recreating and be aware that a performer will deliver the lines. The translator, then, must take into account both the pragmatic and the semantic expressiveness of the word and remember that they are always at work simultaneously. I will take examples both from my personal experience and from remarkable cases in point of how a good translation may affect an audience reception of a foreign play and I will show that it is primarily through a pragmatic approach that it is possible to obtain an awareness of what is the most appropriate way of rendering the original text.
\end{abstract}

Keywords: Theatre translation. Contemporary English drama. Pragmatic approach,. Holistic context.

One possible way of looking at translation activity could be to regard it as a mirror which is held up to accurately represent a 
source text. The reflected image will never look exactly the same as the original; nevertheless, it is to be trusted as being a true copy of it. The best achievement, then, we can aim at when translating is to "say almost the same thing," as Umberto Eco stated with the title in his extensive study on translation. ${ }^{1}$ Although this process of rendering a text from a language into another may lead to the conclusion that it is a task not to be fully fulfilled, in a paradox, I would like to state that this is far from being an absolute failure. We should read it in the light of the aesthetics of failure Samuel Beckett coalesced in Worstward Ho: if language by definition fails to translate thought still it is complicit in representing $\mathrm{it}^{2}$; therefore, if we are aware that it is not possible to render "faithfully" an original text into another language, all the same the translation reifies this impossibility. Although we know we are doomed to failure, this awareness must generate an expansion in creative activity. After all, as Beckett wrote, all an artist can do is to reiterate his/her attempts: "Ever tried. Ever failed. No matter. Try again. Fail again. Fail better." ${ }^{3}$ Since I first read this, it has become my motto as a translator. Theatre translation is usually seen as a more complex dimension of literary translation since the text being translated is thought to be just one of the elements of theatre discourse that one has to render in a different language. Besides, theatre is a mirror of the world, a mirror that not only reflects the verbal utterances but also actions, gestures, silences and the whole apparatus that goes together with them. That is why in translating for theatre the intrinsic impossibility of translation becomes an even more complicated process. However, as Anne Ubersfeld wrote in Reading Theatre, "In theatre the impossible reigns, theatre works with the impossible, and is made to express the impossible." 4 As a matter of fact, the rhetorical figure prevailing in theatre is the oxymoron which, for instance, shows the contradictions rooted in the characters - and in the situation they have to cope with - that make theatrical actions move forward on the stage. ${ }^{5}$

"Drama, as an art form, is a constant process of translation," as Reba Gonstand observed, and "from original concept to script 
(when there is one), to producer's/director's interpretation, to contribution by designer and actor/actress, to visual and/or aural images to audience response [...] there may be a series of subsidiary processes of translation at work." 6 In the dynamic recreation of a source text, then, with the awareness of the peculiarity of a language stylistics, cultural differences, and different theatrical conventions we should also keep in mind that in the consequential act of staging a play there are always different kind of translations involved. We can then assert the written text is incomplete since it is indissolubly linked to performance - as Uberfeld stated it is "troué," it has gaps ${ }^{7}$ - so we must fill in the blanks by finding answers in the performance itself, and only this combination gives us a complete play-text, a text mediated by the two elements. The distinction between written text and performance is an artificial one since, she argues, the two cannot be separated. Their relationship is not just that of a semantic equivalence because the performance cannot be perceived as a mere "translation" of the text. It would be dangerous to assume that the expression of a context results from a conversion from the linguistic sign system to a system of performance/stage signs; as a matter of fact, this attitude may lead to the conclusion that there is only a single correct way to interpret it. We know that different performances give us different readings of a text, and it is not only a matter of a director's choice because it is even impossible to reproduce the same text night after night in performance: we will notice different gestures, slight changes in intonation, different answers to and from the different audience present in the auditorium. As a consequence, we can infer that there isn't a definitive written text. However, incomplete though it may be, it is only through its structure that the performative aspect can be expressed and it is the raw material on which the translator has to work. As Susan Bassnet remarked, "it is with the written text, rather than with a hypothetical performance, that the translator must begin." 8 Nevertheless, the translator also knows that the translation cannot maintain the text in its original state because 
it is intended for a future situation of enunciation staged for an actual target audience. Therefore, as Patrice Pavis commented, the translation takes place at the intersection of the situations of enunciation in a given target language and culture, and even though we possibly have only a vague notion of a given culture inevitably it is within the aspects which determine its identity that a translation takes place:

Translation is this undiscoverable mythic text attempting to take account of the source text - all the while with the awareness that such a text exists only with reference to a source-text-to-be-translated. Added to this disturbing circularity is the fact that theatre translation is never where one expects it to be: not in words, but in gesture, not in the letter, but in the spirit of a culture, ineffable but omnipresent. ${ }^{9}$

When translating a play, the translator should always adapt the text he or she is recreating for performance and be aware that a performer will deliver the lines. Clearly enough then, his/her task is not simply to decode a text into a different language creating a linguistic equivalence. Actually, I believe there's no such thing as a literal translation because it will always be the product of someone who re-creates a text for performance to grant an audience access to a specific text. After all, a translation consists in changing a text to suit different needs. The translator's craft will only produce a transitory text whereas the work of art is timeless. The socio-cultural context so unconditionally clear for its contemporary audience and fellow citizens is yet empty or just ornamental for historical latecomers or outsiders: we need to regain the lost meaning by substituting source text details with elements that are communicative for us in the same way as the ones in the original play were for the source audience. ${ }^{10}$ The translator, then, must sense both the pragmatic and the semantic expressiveness of the word observing the distinct dramatic traditions of the languages from and into which s/he is translating. A theatre 
translation above all has to function within the immediate context of performance - without annotations or editorial commentary. Often the translator is also requested to meet the expectations of a particular audience and to provide familiar details for it in the new version; this strategy may lead to adaptations that have a great distance from the original and possibly suffer the heavy influence of the director or of the producer imposing their own choices. So, which are the strategies that must be developed for dealing with what seems to be untranslatable or unspeakable? What we need is probably a combination of processes of adaptation, interpretation, paraphrasing, contemporization, and most importantly understanding and collaboration. It is this holistic context, as Andras Nagy suggests, rather than a distinct text that poses the real challenge to the translator for performance. ${ }^{11}$ And its observance probably grants a "true respect" for the text being translated. ${ }^{12}$ If we look again at the simile of the mirror, the peculiar translation adapted to function in one country could be just a fragment of the shattered mirror in which the original image was reflected. It follows that a translated theatre text emerges as a variety of reflections and images generated by the historical context and by the cultural environments, as if we were looking back at the original through a kaleidoscope.

Not only should the ideal recreation of the original play convey its linguistic characteristics and its cultural references, but it should also aim to reproduce or to "wisely" interpret the aspects arisen from the collaborative process that produced it. This is particularly true for those plays which playwrights have workshopped in close collaboration with directors and actors. In translation there seems to be a relation of asymmetry with the original text because it is not distorted but deliberately re-crafted to address the ultimately ephemeral moment in which it is to be performed. ${ }^{13}$ The translator, as re-creator of the text, should be acknowledged as a key figure within the collaborative process of production comparable to the role of the playwright, of the dramaturg and of the director. Each new translation, like each new production, involves a distinctive set of artistic and pragmatic choices; the result cannot claim to be 
definitive, it works only until the voice through which it speaks has resonance to its target audience. The translator has rarely been acknowledged as a creative figure integral to the process of production, a creative figure in the shaping of the performance text through rehearsals and production meetings. As a matter of fact, the current practice in Italy, for instance, when translating contemporary texts is to commission a translation to academics or experts in a given foreign language to obtain a raw text which can then be reworked in a new version by a known playwright or dramaturg but, more often, by the director him/herself even though they do not always have an appropriate competence in the source language (this practice often arises more than a suspicion on a contemptible habit to plagiarize previous translations of the text in question). With classics, a previously published translation is usually readapted by the director him/herself. This is true only when the translator is not a prominent figure in the cultural scene or when s/he is not the official translator of a particular playwright. Probably this happens because new versions or adaptations seem to have a higher status than translations. ${ }^{14}$ Perhaps the implication has been that an adaptation requires a level of dramaturgical skill and creative vision, which, in a mere translation, are solely attributable to the original playwright? This provocative suggestion conveys some truth: often translators are not trained to translate for theatre. In most of the cases, they only have a literary academic education.

\section{A case in point}

My first personal experience of collaboration in the creative process of translation is a good case in point to go further in this exploration. The text I'll take into consideration is Philip Ridley's The Pitchfork Disney. I first came across it while translating Aleks Sierz's In-Yer-Face Theatre into Italian. This was a really fruitful experience since it allowed me to literally plunge into contemporary British drama and gain a deep knowledge of the theatrical innovation 
that took place at the end of the 20th century. Moreover, Aleks' prompt answers to my queries were crucial in giving me confidence to complete the translation with the knowledge I was serving his purpose. An author's tips are always useful for a translator - it doesn't matter which genre of text you are working on.

I was fascinated with Ridley's play, one of the first to introduce a new sensibility on London theatre scene. ${ }^{15}$ When in 2005, together with the company I formed with fellow former students of the acting school I attended, we were asked to stage a play in a theatre festival in Spoleto I didn't hesitate to propose The Pitchfork Disney. We could have worked with an existing translation of the play, published as The Killer Disney, but I didn't like it because of its literariness, its lack of theatrical rhythm, elaborate syntax and its slavishness; therefore, I decided to translate it again. My professional education as a translator is mainly academic but I've been taught by professors who often worked in theatre as translators or directors. Moreover, being myself an actor as well, I could approach the written text with an ear for performance. When a very first draft of the translation was ready I asked a playwright (who studied acting together with me) to read it and make the adjustments she thought necessary. She was probably too creative. As a result she re-imagined the text through the eye of the female protagonist and heavily changed it: I had to struggle to restore what I thought was necessary. Notwithstanding, she also introduced some very interesting solutions from a semantic point of view and the text acquired a colloquial dimension that improved the directness of the telling of the story. Eventually, she played the female protagonist having become perfectly tuned with that role. I was playing the male leading part, so working together helped us a lot in pitching the translation at the "right" register. In spite of the play being set in London's East End, it lacked any true concern with dialect and class inflected language. We just differentiated the childish language of Haley and Presley, the two brothers we were playing, from a kind of jargon used by Cosmo, the entertainer, a character which is their exact opposite. 
The process of adaptation was revealing of many aspects of theatrical interpretation, so action became more talkative than originally planned. Besides, instead of a naturalistic setting, the director chose to stage it with just a few elements of furniture: the skeleton of a door and of a window, an old fridge and a couple of chairs which were all constantly moved on scene to stress changes in the developments of characters' perspective.

I was aware of the audience we would have played for. Usually at festivals you have a theatre-wise public, who has extensive knowledge of the theatre scene. So, I decided not to translate some elements (i.e., the title and the names of the protagonists) and references to the Britishness of the British play (i.e., tea cups). Furthermore, after the recent wave of new writing coming from the UK, a trend had been set for British plays which we hoped to exploit commercially. Nonetheless, I didn't believe that totally relocating the alien source material within the cultural experience of the target audience would have helped in the comprehension of the play. Whether a process of domestication represents an undue betrayal of the source, or due recognition of the target is still a matter of opinion. The decision to relocate a play is arguably more consequential with a text for performance but still it is a decision to be taken with a scrupulous attention. And the translator must be well aware of the constant process of redefinition of the contemporary target culture. After playing open air in Spoleto, in a charming 14th-century cloister and in front of an audience of about three-hundred, we presented The Pitchfork Disney in Rome in a small fringe theatre, the classical black-box, for a three-week run for an average audience of about thirty. As a consequence, we had to re-work the translation. It's really different when you pronounce a line and you have to walk for seven or eight metres or if you just have to take two or three steps. The text changes as the space changes as the acting changes. I tried to modulate the words into a tone more suitable for that space, to condensate lines in order to get a more essential language. Also, together with the director, we cut from the text. We thought some of the extensive monologues 
would have not been "tolerated" by the different audience in that claustrophobic auditorium. We then moved in a bigger theatre in Rome and we also went on a short tour but we didn't significantly change the text after that.

The above mentioned experience strengthened in me the belief that an ideal translator for theatre not only must be trained at university to gain linguistic and cultural competence but also at theatres or academies of dramatic arts. After all, a translator should write for actors like playwrights do. According to this view - which in some way is similar to what Robert Corringan stated as far as in the Sixties about translators being trained in the practice of theatre: "Without such training, the tendency will be to translate words and their meanings. This practice will never produce performable translations"16 - I recently promoted an agreement between Italy's National Academy of Dramatic Arts "Silvio d'Amico" and "Sapienza" University of Rome where I teach courses of Translation from English into Italian and of English Drama (the agreement was compiled and signed during the 2009/2010 academic year by Professor Isabella Imperiali, on behalf of "Sapienza" University of Rome, and by Maestro Lorenzo Salveti, Director of Accademia D'Arte Drammatica "Silvio d'Amico"). Students who follow my and Professor Imperiali's courses of Translation for Theatre will be allowed to put into practice what they study, and shape their translations through a collaboration with students of acting and directing courses from the Academy. Students from the University are supposed to enter the rehearsal room of the studio theatre of the Academy with a first draft of their translation (done under my supervision) and after a couple of weeks of rehearsals with actors, directors and teachers, they should be able to come out with a translation that would answer the requirements of the abovementioned holistic context. Working in workshops and interacting face to face will enable students to acquire an awareness of a comprehensive method which wishfully they will later apply in their professions. At the beginning of November 2010 we had the chance to put into practice what we had envisaged with the 
agreement when the boards of the academy and of the university agreed to fund a workshop on Martin Crimp's plays. I chose to work on The Treatment, a challenging text Crimp wrote for the Royal Court Theatre in 1993 whose translation hadn't been published yet in Italy. The Treament is a play set in New York and uses the metropolis as a convention in the same way some Jacobean playwrights used Venice to distance their own society from its decadence; this city drama, as Sierz remarked, is a "cry of dismay at how art is judged by commercial rather than aesthetic criteria." 17 I selected five students from my MA course on translation and asked them to translate it. I assigned to each of them a few scenes, and when they had a first draft of their translation we met and read it aloud: in this way they were able to confront their solutions and to conform recurrent terms, names, and to convey metaphors and images in the same way. The need to conform the five different sections of the translated text (one for each translator) was indeed a good way to reflect on the playwright's style. I obviously corrected mistakes and proposed suggestions for difficult passages so as to find together a valid solution. What was more difficult was to make them aware of the necessity to find a definite way to characterize characters' idiolect and to maintain it throughout. We managed to overcome this difficulty through a deep analysis of the text and a detailed study of characters.

An important aspect of the project was the meeting we organized for the participants to the workshop with Martin Crimp and theatre critic Aleks Sierz. As a matter of fact I believe it is extremely useful, when translating a text of a contemporary living author, to have the possibility to ask questions to him/her. And both Crimp and Sierz were very generous with students. Moreover, Crimp translated several plays into English so his suggestions were really helpful and specific. He thinks there are different ways to approach a translation for theatre. It is possible to have a straight translation from a language the translator understands, whose aim is to provide a window on the original text. In this case, the only concern for the translator is that the translation will be actable; s/he doesn't 
want to appear but just wants to serve the writer, the play and also the production. We can infer two different possibilities to proceed in this instance from two translations Crimp was commissioned to do. When in 1997 he translated Eugène Ionesco's Les chaises for the Royal Court Theatre, director Simon McBurney didn't want the play to appear as a French artefact, so he suppressed all the references to that cultural environment; whereas, Crimp kept them when translating Ionesco's

Rhinocéros (Royal Court Theatre, 2007) since director Dominic Cook wanted to recreate a world of France in the Fifties, so he did want this artefact. It is also possible to fashion a transparent straight translation based on an intermediate text from a language the translator doesn't understand. Crimp doesn't like this option because he believes it doesn't allow you to get the thrill of the original language. He compares it to shaving in front of a steamed mirror: you risk to cut yourself really badly. Finally, a translator can adapt, rewrite, renovate and reshape a text so as to tailor it for an audience who speaks a different language and lives in a different country and in a different time. Crimp followed this course of action when he translated Molière's Le misanthrope (Young Vic, 1996; Comedy Theatre, 2009), because he believes that, for instance, the contingent satire in The Misanthrope would otherwise get lost. He also produced a pared-down version of Anton Checov's The Seagull (National Theatre, 2006). In the final text 19th-century references were either replaced with equivalent or altogether removed and it was also stripped from 19th-century theatrical conventions - such as monologues and asides. Crimp's version was obviously shorter but also more vibrant, fresher than the original, renovated as an old painting could be after a process of restoration. Translating for performance within a given context requires a sensitivity to the various agendas in operation in both the source and target cultures - whether in terms of state censorship or, at a more pragmatic level, institutional production policies. As cultural advocate, the translator may refuse to relocate the source text for fear of neutralising its cultural identity. For instance, when 
in 2009 the Belarusian company Belarus Free Theatre came to play in Italy (at Vie Scena Contemporanea theatre festival in Modena), I was asked to revise and adapt the translations of a couple of their plays because I had been to Minsk to work with them, knew their plays and particularly their peculiar condition of dissidents to the totalitarian regime of Alexander Lukashenko. In that case there was no use in domesticating their plays: what had to emerge was an awareness of what is life in Belarus under the abuses of a dictatorship which was to be conveyed with absolute consistency.

\section{Conclusion}

Intercultural communication always depends on varied and complex processes, which influence not only the production of a theatre translation but also its distribution and reception by a diverse target public. It is mainly with practice and with a pragmatic approach that is possible to get an awareness of what is the most suitable solution according to the place and to the persons involved. However interesting and stimulating are the two polarized theoretical constructs of performability and readability that have animated the theoretical debate about translation for theatre, when applied to actual translations and theatrical performances they seem to share a weakness in their prescriptive approaches. In particular, I believe it is rather singular to speak about readability for a text conceived and written to be spoken and performed. Usually, in the process of creation, the words that constitute the language of the play are first heard by the playwright as his or her characters start to come to life, he or she then writes them down to be spoken by actors and/or actresses who in turn deliver them for a given audience who listens to them. This is roughly the process of what we call theatre which is mainly made of a combination of utterances and listenings even though it makes use of written words. To be defined stageworthy, a translation should flow like the original text flows. And this is what a theatre translation should aim to do and 
what, in my opinion, defines the good sense and sound judgment a wise translator should have. Having no wish, then, to impose any particular rule or method on translation studies, I just wish translators to fail in the best possible way.

\section{Notes}

1. See, Umberto Eco, Dire quasi la stessa cosa, esperienze di traduzione (Milano: Bompiani, 2003).

2. C. J. Ackerley, S. E. Gontarski, eds., The Faber Companion to Samuel Beckett (London: Faber \& Faber, 2006), 652-53.

3. Samuel Beckett, Worstward Ho (London: John Calder, 1983), 7.

4. Anne Ubersfeld, Reading theatre (Toronto: University of Toronto Press, 1999), 190.

5. See Ubersfeld, Reading theatre, $190 \mathrm{ff}$.

6. Reba Gonstand, "Verbal and non verbal communication: drama as translation," in O. Zuber ed., The Langage of Theatre. Problems in Translation and Transposition of Drama. (Oxford and New York: Pergamon Press, 1980), 1.

7. See Ubersfeld, Reading theatre, xvi; 10.

8. Susan Bassnet, "Ways through the Labyrinth: Strategies and Methods for Translating Theatre Texts," in Theo Hermans, ed., The Manipulation of Literature (London: Croom Helm; New York: St Martin's, 1985), 102. 
9. Patrice Pavis, "Problems of Translation for the Stage: Intercultural and Post-Modern Theatre," in Peter Holland, Hanna Scolnicov, eds., The Play Out of Context: Transferring Plays from Culture to Culture (Cambridge: Cambridge University Press, 1989), 42.

10. See Andras Nagy, "A samovar is a samovar: Hopes and failures of the author as the object and subject of translation," in Moving Target, Theatre translation and Cultural Relocation, ed. by Carole-Anne Upton (Manchester: St. Jerome Publishing, 2000).

11. Andras Nagy, “A samovar is a samovar," 152.

12. The importance of an "holistic" approach was advocated by Pavis since at least 1989, when for instance he wrote: "The translator is a dramaturg who must first of all effect a macrotextual translation, that is, a dramaturgical analysis of the fiction conveyed by the text. S/he must reconstitute the plot according to the logic that appears to suit the action, and so reconstitute the artistic totality," in Patrice Pavis, "Problems of Translation for the Stage: Intercultural and Post-Modern Theatre," 27.

13. See Hale and Upton, "Introduction," in Moving Target (Manchester, UK \& Northampton MA: St. Jerome Publishing, 2000).

14. See for instance what Maria Delgado states in Phyllis Zatlin, Theatrical translation and film adaptation: a practitioner's view (Clevendon: Multilingual Matters, 2005), 26.

15. Aleks Sierz, In-Yer-Face Theatre: British Drama Today. (London: Faber \& Faber, 2001), 40-47.

16. Robert W. Corrigan, "Translating for Actors," in W. Arrowsmith and R. Shattuck eds., The Craft \& Context of Translation. (Austin: University of Texas Press, 1961), 100.

17. Aleks Sierz, The Theatre of Martin Crimp. (London: Methuen Drama, 2006), 40. 


\section{References}

Aaltonen, Sirkku. Time Sharing on Stage: Drama Translation in Theatre and Society. Clevendon: Multilingual Matters, 2000.

Corrigan, Robert W. "Translating for Actors," in W. Arrowsmith and R. Shattuck, eds., The Craft \& Context of Translation. Austin: University of Texas Press, 1961.

Elam, Keir. The Semiotics of Theatre and Drama. London: Routledge, 1980.

Johnston, David. Stages of Translation. London: Absolute Classics, 1996.

Patrice, Pavis. Dictionaire du Théâtre. Paris: Armand Colin, 2002 [1980].

Scolnicov H. and Holland P. The Play out of Context: Transferring Plays from Culture to Culture. Cambridge: Cambridge University Press, 1989.

Venuti, Lawrence. The Translator's Invisibility. London: Routledge, 1995.

Wechsler, Robert. Performing Without a Stage: the Art of Literary Translation. North Haven: Catbird Press, 1998.

Zuber, Ortrun. The Languages of Theatre:. Problems in the Translation and Transposition of Drama. Oxford and New York: Pergamon Press, 1980. 